\title{
Bioanalysis
}

\section{Bioanalytical derivatization: is there still room for development?}

\begin{abstract}
"...derivatization in its simplest and most effective form will likely stay the basis of rapid and cheap color test reactions for drug substances, diagnostic markers and many more."
\end{abstract}

Keywords: derivatization $\bullet$ detection $\bullet$ fluorescence $\bullet \mathrm{GC}(-\mathrm{MS}) \bullet \mathrm{LC}(-\mathrm{MS}) \bullet$ separation mark real progress, and if not, which new fields are there to be discovered.

\section{General aspects}

Some general aspects apply for almost all derivatization-based strategies: kinetic aspects, in other words, does a derivatization reaction proceed equally good at different concentrations, particularly when analyte concentrations become very low $(\mathrm{pM}-\mathrm{nM})$; pre- versus post-column derivatization, in other words, how does an analytical platform in a precolumn approach react to the usually very high concentration(s) of reagent(s); reaction completeness, in other words, does a reaction proceed smoothly to a single derivative and would considerable amounts of underivatized analyte affect the analytical process? All these aspects are important to the development and application of analyte derivatization. Therefore, scientists working with derivatization should understand the significance of the listed issues, the nature of the reaction and the possible pitfalls related to the employed approach in order to allow critical assessment of obtained results.

\section{Derivatization for GC}

GC is certainly one of the most prominent fields in which derivatization plays a crucial role for stabilizing analytes and transforming them into volatile derivatives, ultimately allowing their analysis. Over the last decades, numerous derivatization reagents have been described [5]. Some reagents have become widely accepted and proved their applicability in numerous examples. In this context, undoubtedly methoxyamination

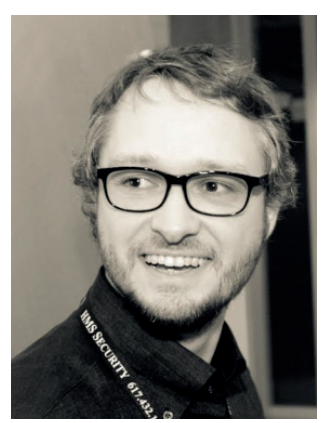

Martin Giera Center for Proteomics \& Metabolomics, Leiden University Medical Center, Albinusdreef 2, 2300RC Leiden, The Netherlands m.a.giera@lumc.nl 
and silylation have to be named [6,7]. Other prominent examples include pentafluorobenzyl bromide, chloroformates and methylation reactions. Overall, certain reagents in combination with either electronionization or negative-ion chemical ionization have become widely accepted allowing for the analysis of a very broad range of substances [5]. Hence, it seems difficult presenting real progress for novel derivatization approaches in GC-MS analysis.

\section{Derivatization for LC}

Unlike for GC, derivatization reactions for LC have mainly been developed for detection purposes. Classic examples include $o$-phthalaldehyde or ninhydrine for the fluorescence-based detection of amino acids [8]. However, particularly during the last decade, many reagents have been developed aiming at enhancing detectability by enhancing ionization efficiency during electrospray ionization (ESI) [9]. Many of these reagents aimed at introducing permanent charges into the analyte of interest. In light of an empirically difficult detection of negatively charged analytes, many of these reagents also tried to inverse the charging so that the analytes could be detected in the positive-ion ESI mode (ESI+) after derivatization (e.g., carboxylic acids). Nevertheless, unlike for GC-MS, derivatization is usually not a necessary prerequisite for LC-MS. Moreover, instrumental developments have rendered negative mode ESI (ESI-) as sensitive as ESI+. Therefore, novel developments in derivatization for LC-MS analysis might be useful for neutral, hard to ionize components (e.g., sterols, small aldehydes) or for the stabilization of certain analytes such as keto-carboxylic acids. However, the analyst should always ask himself whether the process of derivatization yields a real advantage in terms of separation and/or detection and if more simple analytical approaches might not be pursued instead.

\section{"GC is certainly one of the most prominent fields} in which derivatization plays a crucial role..."

\section{Chiral derivatization}

Of importance to GC-MS and LC-MS is the transformation of enantiomers into separatable diastereomers using chiral reagents. The most well-known example might be the use of Mosher's acid chloride in GC-MS, which has also been used in assisting NMR-based determination of absolute stereochemistry $[5,10]$. In the light of chiral metabolomics, it will be important to further our capabilities of group-specific chiral derivatization and overcome related issues like substance identification, determination of absolute stereochemistry and others [11].

\section{Isotopic labeling strategies}

An interesting concept, facilitating quantitative GC-MS and LC-MS analysis, is stable isotope encoded derivatization. Although this concept has been described for the use in metabolite analysis [12], it particularly has become widely used in quantitative proteomics where its main application is known as isobaric tagging for relative and absolute quantification (iTRAQ) [13]. The development of these approaches can certainly be indicated as a prime example for the usefulness of derivatization in bioanalysis. Further developments in this field can be expected, especially because innovations overcoming quantification issues, particularly in LC-MS are still urgently needed.

\section{Other derivatization-based analyses}

A field where much work remains to be done is the derivatization based (quantitative) fluorescence or MS-based imaging of metabolites in cells and tissues. While particularly MS imaging (MSI) approaches are already capable of showing, for example, the distribution of drug molecules in animal studies, particularly low abundant and fragile analytes remain elusive. In these latter cases, bioanalytical derivatization might very well play an important role enabling MSI of certain metabolites. However, spatial distribution, side reactions and other problems, such as matrix effects, will need to be overcome. An example for endogenous amines was recently presented by Manier et al. [14].

Finally, the outstanding importance of thin layer chromatography (TLC) in combination with derivatization has to be emphasized. TLC remains a key technique in the organic chemistry laboratory enabling fast and cheap reaction control.

\section{Conclusion \& future perspective}

Derivatization is an inevitable tool in numerous bioanalytical approaches. However, the analytical chemist should always critically evaluate whether or not it is really necessary. Even though many derivatization reactions are well described, the process of derivatization introduces additional steps in the bioanalytical protocol, possibly causing bias or jeopardizing study outcome, especially when the analyst is insufficiently aware of the nature and purpose of the derivatization process. Although generally of value, the further enhancement and development of derivatization reagents for GC(MS) seem difficult. With the reactions named earlier, a very wide range of analytes is covered using elegant and well-established labeling strategies.

In case of LC-MS, especially charge-reversal derivatization seems redundant as today's mass spec- 
trometers have equal sensitivity in ESI+ and ESI- mode. Particularly for LC-MS approaches, the necessity of derivatization should be critically evaluated. Possible improved detection and separation should be weighed against the necessary efforts and potential artifact formation. Thus, the possibility of using alternative approaches should be considered. Especially when analytes are present at relatively high concentrations, derivatization does not always seem justified. A prominent example is the derivatization-based LC-MS analysis of fatty acids where numerous derivatization approaches have been presented while basically these analytes are present at fair amounts in biological samples and can be readily separated and detected using LC-MS in the ESI- mode.

Personally, I see real possibilities for new developments in the fields of metabolite imaging as well as quantitative techniques. Imaging-based analysis might include fluorescence or MS-based imaging of metabolites. In this case, many analytical pitfalls will have to be overcome. Furthermore, metabolite quantification should be achieved. However, as derivatization enabled many bioanalytical developments, it might very well be of high value to MSI and other imagingbased approaches. In the case of quantitative bioanalysis, derivatization has had a strong influence on important developments in the last decades and hopefully more reagents, reactions and concepts will be developed helping us to overcome fundamental bioanalyti-

\section{References}

1 Wieland H, Scheuing G. Die fuchsin-schweflige säure und ihre farbreaktion mit aldehyden. Ber. Dtsch Chem. Ges. A/B 54(10), 2527-2555 (1921).

2 Dalgliesh CE, Horning EC, Horning MG, Knox KL, Yarger K. A gas-liquid-chromatographic procedure for separating a wide range of metabolites occuring in urine or tissue extracts. Biochem. J. 101(3), 792-810 (1966).

3 Oliver SG, Winson MK, Kell DB, Baganz F. Systematic functional analysis of the yeast genome. Trends Biotechnol. 16(9), 373-378 (1998).

4 Giera M, Wuhrer M. Recent developments in clinical omics. Chromatographia 78(5-6), 305-306 (2015).

5 Zaikin V, Halket J. A Handbook of Derivatives for Mass Spectrometry. IM Publications, Chichester, UK (2009).

6 Eric Chun Yong C, Kishore Kumar P, Jeremy KN. Global urinary metabolic profiling procedures using gas chromatography-mass spectrometry. Nat. Protoc. 6(10), 1483-1499 (2011).

7 Kloos D, Lingeman H, Mayboroda OA, Deelder AM, Niessen WMA, Giera M. Analysis of biologically-active, endogenous carboxylic acids based on chromatography-mass spectrometry. Trends Anal. Chem. 61(0), 17-28 (2014).

8 Rigas P. Post-column labeling techniques in amino acid analysis by liquid chromatography. Anal. Bioanal. Chem. 405(25), 7957-7992 (2013). cal issues such as for example matrix effects or analyte losses during extraction and analysis.

Ultimately, derivatization in its simplest and most effective form will likely stay the basis of rapid and cheap color test reactions for drug substances, diagnostic markers and many more. Particularly, as simplicity and ingenuity frequently go together, it will be very interesting to see to which extent clinical biomarkers might be translated into simple color reaction based tests or to which extent our ubiquitous mobile phones with their ever-increasing processor and camera power might be used to read out derivatization reactions for diagnostic purposes or the identification of drug substances.

\section{Acknowledgements}

The author would like to thank WMA Niessen for countless fruitful discussions concerning derivatization during the last years and his constructive criticism concerning this manuscript.

\section{Financial \& competing interests disclosure}

The author has no relevant affiliations or financial involvement with any organization or entity with a financial interest in or financial conflict with the subject matter or materials discussed in the manuscript. This includes employment, consultancies, honoraria, stock ownership or options, expert testimony, grants or patents received or pending, or royalties.

No writing assistance was utilized in the production of this manuscript.

9 Santa T. Derivatization reagents in liquid chromatography/electrospray ionization tandem mass spectrometry. Biomed. Chromatogr. 25(1-2), 1-10 (2011).

10 Hoye TR, Jeffrey CS, Shao F. Mosher ester analysis for the determination of absolute configuration of stereogenic (chiral) carbinol carbons. Nat. Protoc. 2(10), 2451-2458 (2007).

11 Nagao R, Tsutsui H, Mochizuki T et al. Novel chiral derivatization reagents possessing a pyridylthiourea structure for enantiospecific determination of amines and carboxylic acids in high-throughput liquid chromatography and electrospray-ionization mass spectrometry for chiral metabolomics identification. J. Chromatogr. A 1296, 111-118 (2013).

12 Yang W-C, Adamec J, Regnier FE. Enhancement of the LC/MS analysis of fatty acids through derivatization and stable isotope coding. Anal. Chem. 79(14), 5150-5157 (2007).

13 Chahrour O, Cobice D, Malone J. Stable isotope labelling methods in mass spectrometry-based quantitative proteomics. J. Pharm. Biomed. Anal. 113, 2-20 (2015).

14 Manier ML, Spraggins JM, Reyzer ML, Norris JL, Caprioli RM. A derivatization and validation strategy for determining the spatial localization of endogenous amine metabolites in tissues using maldi imaging mass spectrometry. J. Mass Spectrom. 49(8), 665-673 (2014). 